\title{
Esquemas de Alocação de Canais e Geração de FWM em Sistemas DWDM: Estudo Comparativo
}

\author{
Paula Brandão Harboe, Edilson da Silva e José Rodolfo Souza
}

\begin{abstract}
Resumo-Este trabalho apresenta um estudo comparativo de como diferentes esquemas de alocação de canais baseados em espaçamento não uniforme (US) afetam a geração de FWM em sistemas ópticos multicanal com fibra de dispersão deslocada. A influência da banda passante de filtros ópticos também é investigada e a largura de banda e relação sinal-ruído (SNR) do sistema são usadas como figuras de mérito. Os resultados indicam que o esquema US cíclico com intervalo não uniforme (USC-NU) provê bom compromisso entre largura de banda ( 20nm) e SNR (> $20 \mathrm{~dB})$, considerando um sistema de 24 canais com filtro óptico de 25 GHz.
\end{abstract}

Palavras-Chave - Sistemas DWDM, Four-Wave Mixing (FWM), Esquemas de Alocação de Canais, Relação Sinal-Ruído.

Abstract - This paper presents a comparative study of how different channel allocation schemes based on non-uniform spacing (US) affect the generation of FWM in optical systems based on dispersion-shifted fibers. The paper also investigates the influence of the pass-band of optical filters on this process, taking the overall system bandwidth, and signal-to-noise ratio as the major figure of merit. The results indicate that the US scheme with non-uniform interval (USC-NU) represents a good compromise between bandwidth $(\sim 20 \mathrm{~nm})$ and SNR $(>20 \mathrm{~dB})$, considering a 24-channel system together with a $25 \mathrm{GHz}$ filter.

Keywords - DWDM systems, Four-Wave Mixing (FWM), Channel Allocation Schemes, Signal-to-Noise Ratio (SNR).

\section{INTRODUÇÃO}

Sistemas multicanal de transmissão óptica de alta capacidade são fundamentalmente limitados pelo efeito não linear de mistura de quatro ondas (FWM), responsável pela geração de novas frequências ópticas (ou produtos FWM) e interferência (crosstalk) entre canais [1]. A eficiência de geração de FWM depende do esquema de alocação de frequências dos canais e do espaçamento entre eles, assim como do tipo de fibra empregado.

Em trabalhos recentes [2]-[6], os autores desenvolveram um simulador WDM versátil e robusto para a análise das penalidades impostas por FWM em fibras monomodo(G.652), de dispersão deslocada (G.653) e de dispersão deslocada não nula (G.655), considerando espaçamentos uniforme (ES) e não uniforme (US) entre canais.

Paula B. Harboe e Edison da Silva - Departamento de Engenharia de Telecomunicações, Universidade Federal Fluminense (UFF), Rua Passo da Pátria 156 Niterói - RJ. E.mail: Paula@telecom.uff.br.

José Rodolfo Souza - Departamento de Eletrônica e Telecomunicações, Universidade do Estado do Rio de Janeiro (UERJ), Rua São Francisco Xavier, 524 - Bloco A, Rio de Janeiro - RJ. E. mail: jrsouza@uerj.br.
O modelo utilizado supõe bombeio sem depleção e permite avaliar o descasamento de fase, potência de FWM e a relação sinal-ruído (SNR) de sistemas com elevado número de canais e espaçamentos ultrafinos, de até $6,25 \mathrm{GHz}$. Os principais resultados indicam que a manutenção de valores aceitáveis para a SNR do sistema depende, fundamentalmente, da potência por canal, do espaçamento entre canais e do tipo de fibra empregado. Em particular, o mecanismo de FWM é acentuado quando os canais são alocados na região de zero de dispersão da fibra (no comprimento de onda $\lambda_{\mathrm{ZD}}$ ), pois a condição de casamento de fase é praticamente satisfeita. Além disto, na alocação ES, todos os produtos FWM que ocorrem na banda do sistema coincidem, em frequência, com algum dos canais, dando origem à severa interferência. Por outro lado, na alocação US, quando a separação de frequências entre quaisquer dois canais de um sistema de WDM é única (não se repete), nenhum produto FWM coincide, em frequência, com qualquer um dos canais. Esta idéia baseia-se no conceito de régua de Golomb [7] e, embora reduza as penalidades impostas por FWM, aumenta a largura da banda total ocupada pelo arranjo de canais. Em sistemas DWDM com elevado número de canais, o problema se torna ainda mais severo, pois é necessário alcançar larguras de banda totais tão estreitas quanto possível devido à limitada faixa de frequências de operação dos amplificadores ópticos.

Em anos recentes, vários esquemas de alocação não uniforme de canais foram propostos [8],[9]. O presente trabalho tem como objetivo investigar, através de simulação em computador, como tais esquemas de alocação de canais afetam a geração de FWM em sistemas DWDM e se efetivamente contribuem para a redução da mesma. As alternativas investigadas visam aliviar as limitações associadas aos esquemas ES e US. O trabalho investiga, ainda, a influência de filtros ópticos na redução das penalidades de potência impostas pelo mecanismo de FWM.

O trabalho está organizado da seguinte forma: inicialmente, na Seção II, os principais conceitos associados ao mecanismo de FWM são destacados. As quantidades descasamento de fase, potência de FWM e relação sinal-ruído de um sistema WDM são definidas e usadas para implementar (na linguagem FORTRAN 90) o referido simulador WDM. Em seguida, na Seção III, é feita uma comparação entre os desempenhos de diversos esquemas de alocação de canais, medidos pela largura de banda total e pela SNR do sistema. São considerados o esquema de espaçamento uniforme (ES) e variações do esquema baseado em espaçamento não uniforme (US) - aqui denominadas US genérico (US-G), US baseado na régua de 
Golomb ótima (US-OGR), Us cíclico (USC), US cíclico com intervalo uniforme (USC-U) e US cíclico com intervalo não uniforme (USC-NU). Na Seção IV, é investigada a influência de filtros ópticos na redução das penalidades de potência impostas pelo mecanismo de FWM. Conclusões são apresentadas na Seção V.

\section{FUNDAMENTOS DA ANÁLISE}

\section{A. Four-Wave Mixing (FWM)}

Segundo a aproximação de bombeio sem depleção, em que as ondas de bombeio são consideradas muito mais intensas que as ondas de FWM geradas, a amplitude de um produto FWM no final de uma fibra óptica monomodo de comprimento L é escrita como [1]-[6]:

$$
\begin{array}{r}
A_{\mathrm{F}}(\mathrm{L})=\mathrm{i} \frac{\mathrm{n}_{2} \omega_{\mathrm{F}}}{\mathrm{cA}_{\text {eff }}} \boldsymbol{A}_{\mathrm{p}}(0) \boldsymbol{A}_{\mathrm{q}}(0) \boldsymbol{A}_{\mathrm{r}}^{*}(0) \exp \left(-\frac{1}{2} \alpha \mathrm{L}\right) \frac{\exp [(-\alpha+\mathrm{i} \Delta \beta) \mathrm{I}}{\mathrm{i} \Delta \beta-\alpha} \\
\operatorname{com~i}=\sqrt{-1} \text { e } \Delta \beta=\beta_{\mathrm{p}}+\beta_{\mathrm{q}}-\beta_{\mathrm{r}}-\beta_{\mathrm{F}}
\end{array}
$$

$\mathrm{Na}$ equação (1), $\mathrm{A}_{\mathrm{F}}(\mathrm{L})$ é a amplitude do produto $\mathrm{FWM}$ gerado na frequência $f_{F}=f_{p}+f_{q}-f_{r}=\omega_{F} / 2 \pi,(p, q, r=1, \ldots, N$; $\mathrm{p}, \mathrm{q} \neq \mathrm{r}), \mathrm{A}_{\mathrm{s}}(0)(\mathrm{s}=\mathrm{p}, \mathrm{q}, \mathrm{r})$ é a amplitude de um dos $\mathrm{N}$ canais, com frequência $f_{s}$, $\alpha$ é o coeficiente de atenuação da fibra, $n_{2}$ é o coeficiente de índice de refração não linear, c é a velocidade da luz no vácuo, $A_{\text {eff }}$ é a área efetiva do núcleo da fibra, $\beta_{\mathrm{s}}$ é a constante de fase $\mathrm{e}^{*}$ indica complexo conjugado. A equação (1) assume operação CW (Continuous Wave), que representa o pior caso de geração de FWM.

A potência média de um produto FWM na frequência $\omega_{\mathrm{F}}=$ $2 \pi c / \lambda$ é calculada como:

$$
\begin{aligned}
& \mathrm{P}_{\mathrm{F}}(\mathrm{L})=\underset{\mathrm{f}_{\mathrm{r}}=\mathrm{f}_{\mathrm{p}}+\mathrm{f}_{\mathrm{q}}-\mathrm{f}_{\mathrm{F}}}{\sum_{\mathrm{f}_{\mathrm{q}}} \sum_{\mathrm{p}}\left|\mathrm{A}_{\mathrm{F}}(\mathrm{L})\right|^{2}=} \\
& \frac{4 \pi^{2} \mathrm{n}_{2}^{2}}{\mathrm{~A}_{\text {eff }}^{2} \lambda^{2}} \mathrm{~d} \mathrm{P}_{\mathrm{p}}(0) \mathrm{P}_{\mathrm{q}}(0) \mathrm{P}_{\mathrm{r}}(0) \exp (-\alpha L) \mid \frac{\exp [(-\alpha+\mathrm{i} \Delta \beta) \mathrm{L}]-}{\mathrm{i} \Delta \beta-\alpha}
\end{aligned}
$$

A geração de FWM será tão mais eficiente quanto menor o descasamento de frequências e das correspondentes constantes de fase. Na equação (2), d é chamado de fator de degeneração $(d=1$ se $\mathrm{p}=\mathrm{q} \neq \mathrm{r}$ e $\mathrm{d}=2$ se $\mathrm{p} \neq \mathrm{q} \neq \mathrm{r})$ e o termo $\Delta \beta$ representa a diferença das constantes de fase das várias ondas ou, simplesmente, descasamento de fase. Uma expressão analítica para o parâmetro $\Delta \beta$ é obtida a partir da expansão da constante de fase $\beta$ em série de Taylor em torno de uma dada frequência $\mathrm{f}_{0}=\mathrm{c} / \lambda_{0}$, que pode ser a frequência de um dos canais, a frequência do zero de dispersão $\left(\mathrm{f}_{\mathrm{ZD}}=\mathrm{c} / \lambda_{\mathrm{ZD}}\right)$ da fibra ou qualquer outra escolha conveniente.

$$
\begin{aligned}
& \Delta \beta=\beta\left(f_{p}\right)+\beta\left(f_{q}\right)-\beta\left(f_{r}\right)-\beta\left(f_{F}\right) \\
& =\left[\left(\mathrm{f}_{\mathrm{r}}-\mathrm{f}_{0}\right)^{2}+\left(\mathrm{f}_{\mathrm{p}}-\mathrm{f}_{0}\right)\left(\mathrm{f}_{\mathrm{q}}-\mathrm{f}_{\mathrm{r}}\right)-\left(\mathrm{f}_{\mathrm{q}}-\mathrm{f}_{0}\right)\left(\mathrm{f}_{\mathrm{r}}-\mathrm{f}_{0}\right)\right] \frac{2 \lambda_{0}^{2} \pi}{\mathrm{c}} \mathrm{D}(\lambda \\
& -\left(\mathrm{f}_{\mathrm{p}}-\mathrm{f}_{\mathrm{r}}\right)\left(\mathrm{f}_{\mathrm{q}}-\mathrm{f}_{\mathrm{r}}\right)\left[\left(\mathrm{f}_{\mathrm{p}}-\mathrm{f}_{0}\right)+\left(\mathrm{f}_{\mathrm{q}}-\mathrm{f}_{0}\right)\right] \frac{\lambda_{0}^{4} \pi}{\mathrm{c}^{2}}\left[\frac{2}{\lambda} \mathrm{D}\left(\lambda_{0}\right)+\frac{\mathrm{dD}\left(\lambda_{0}\right)}{\mathrm{d} \lambda}\right.
\end{aligned}
$$

$\mathrm{Na}$ equação (3), D é o parâmetro de dispersão da fibra e $\mathrm{dD} / \mathrm{d} \lambda$ é a inclinação (slope) da curva de dispersão. A equação (3) é válida em uma faixa de frequências, em torno de $\mathrm{f}_{0}$, onde o slope é linear, ou seja, onde a dispersão de segunda ordem é constante. De acordo com (3), o descasamento de fase depende do tipo de fibra utilizado e do espaçamento entre canais. A condição de casamento de fase $(\Delta \beta=0)$ é, portanto, praticamente satisfeita em torno de $\mathrm{f}_{\mathrm{ZD}}$.

Para todo e qualquer sistema WDM, garantir que o mesmo tenha uma boa SNR é um fator importante para a recuperação da informação transmitida. Sem conhecer os detalhes dos transmissores e receptores utilizados no sistema óptico, de modo geral, presume-se que os últimos necessitem de SNRs maiores que $20 \mathrm{~dB}$ [10], considerando o ruído como oriundo única e exclusivamente da geração de FWM e que a redução da potência do sinal transmitido pela fibra é devida apenas à atenuação. A SNR é definida como:

$$
\operatorname{SNR}(d B)=10 \log _{10}\left(\frac{P_{\text {sinal }}}{P_{\text {ruído }}}\right)
$$

Para calcular adequadamente a potência de ruído, é necessário identificar todos os produtos FWM pertinentes, ou seja, todos aqueles que estão na banda espectral do filtro óptico responsável pela separação dos canais, e somar suas correspondentes potências. A análise da SNR baseia-se sempre no pior caso encontrado dentre os canais.

As equações (1)-(4) fornecem o formalismo matemático necessário para a investigação do mecanismo de FWM em diversos tipos de fibra óptica e variados esquemas de alocação de canais, que são apresentados na seção seguinte. Estas equações formam a base do simulador WDM implementado na linguagem FORTRAN 90 [2]-[6].

\section{ESQUEMAS DE ALOCAÇÃO DE CANAIS}

\section{A. Espaçamentos Uniforme e Não Uniforme}

No esquema ES, a separação de frequências entre canais adjacentes $(\Delta \mathrm{f})$ é constante; a largura de banda óptica $\left(\mathrm{B}_{\mathrm{ES}}\right)$ para um sistema com $\mathrm{N}$ canais é dada por: $\mathrm{B}_{\mathrm{ES}}=(\mathrm{N}-1) \Delta \mathrm{f}$.

No esquema US, a separação de frequências entre canais é não uniforme. Dois casos distintos são estudados: espaçamento não uniforme genérico (US-G) e espaçamento não uniforme baseado em OGR (US-OGR).

\section{Caso 1: US-G}

A frequência central do i-ésimo canal é dada por: $\mathrm{f}_{\mathrm{i}+1}=$ $\mathrm{f}_{\mathrm{i}}+\Delta \mathrm{f}_{\mathrm{i}}$, com $\Delta \mathrm{f}_{\mathrm{i}}=\mathrm{f}_{\text {ref }}+(\mathrm{i}-1) \Delta \mathrm{F}(\mathrm{i}=1, \ldots, \mathrm{N}-1) . \mathrm{f}_{\text {ref }}$ e $\Delta \mathrm{F}$ são escolhidos arbitrariamente. É comum considerar que a frequência do primeiro canal é nula. A largura de banda óptica é dada por: $B_{U S}=\sum_{i=1}^{N-1} \Delta f_{i}$. Nesta configuração não há garantia de que a separação em frequência entre quaisquer dois canais seja única (não se repita), o que resulta na geração de produtos FWM em frequências coincidentes com as dos próprios canais e, consequentemente, na degradação da SNR. 


\section{Caso 2: US-OGR}

A largura de banda óptica é dividida em slots de mesma largura $\Delta \mathrm{f}$; a frequência central do i-ésimo canal é dada por $\mathrm{f}_{\mathrm{i}}=\mathrm{f}_{\text {ref }}+\mathrm{n}_{\mathrm{i}} \Delta \mathrm{f}(\mathrm{i}=1, \ldots, \mathrm{N})$, onde $\mathrm{f}_{\text {ref }}$ é uma frequência arbitrária de referência e $\mathrm{n}_{\mathrm{i}}$ é um inteiro positivo, denominado "número de slot". Um produto FWM na frequência $f_{F}=f_{p}+f_{q}-f_{r}$ tem um número de slot $\mathrm{n}_{\mathrm{F}}=\mathrm{n}_{\mathrm{p}}+\mathrm{n}_{\mathrm{q}}-\mathrm{n}_{\mathrm{r}}$. Como, neste esquema, $\mathrm{n}_{\mathrm{p}}$, $\mathrm{n}_{\mathrm{q}} \neq \mathrm{n}_{\mathrm{r}}$, então $\mathrm{n}_{\mathrm{F}} \neq \mathrm{n}_{\mathrm{p}}(\mathrm{p}=1, \ldots \mathrm{N})$, o que assegura que nenhum produto FWM coincida com qualquer um dos canais. Isto equivale a dizer que os $\mathrm{N}(\mathrm{N}-1) / 2$ possíveis espaçamentos normalizados entre canais $\left(\mathrm{m}_{\mathrm{i}}=\mathrm{n}_{\mathrm{i}+1}-\mathrm{n}_{\mathrm{i}}, \mathrm{i}=1, \ldots \mathrm{N}-1\right)$ são distintos entre si. Para minimizar a largura de banda, basta fazer com que a soma dos espaçamentos normalizados $\left(\mathrm{m}_{1}+\mathrm{m}_{2}+\ldots+\mathrm{m}_{\mathrm{N}-1}\right)$ seja mínima. Esta solução é baseada no conceito de régua de Golomb ótima (OGR - Optimum Golomb Ruler) [7]. A ordem da régua representa o número de canais e seu comprimento $\left(\mathrm{n}_{\mathrm{N}}-\mathrm{n}_{1}\right)$, a largura de banda normalizada ocupada pelos canais. A largura de banda óptica é dada por: $\mathrm{B}_{\mathrm{OGR}}=\sum_{\mathrm{i}=1}^{\mathrm{N}-1} \mathrm{~m}_{\mathrm{i}} \Delta \mathrm{f}$.

A escolha de $\Delta \mathrm{f}$ deve ser feita de modo a garantir que a largura de banda máxima ocupada pelos canais fique limitada a 30 - $40 \mathrm{~nm}$, que correspondem à largura de banda típica de um EDFA. Em trabalho recente [3], os autores verificaram que, no caso ES, esta condição é facilmente satisfeita, mas o esquema US-OGR limita $\Delta \mathrm{f}$ a valores pequenos: com apenas 11 canais e $\Delta \mathrm{f}=25 \mathrm{GHz}, \mathrm{B}_{\mathrm{OGR}} \sim 15 \mathrm{~nm}$. Para 20 canais e $\Delta \mathrm{f}=12,5 \mathrm{GHz}, \mathrm{B}_{\mathrm{OGR}} \sim 28 \mathrm{~nm}$.

Uma outra questão importante diz respeito à definição da largura de banda do filtro óptico $\left(\mathrm{B}_{\mathrm{F}}\right)$ para o cálculo da SNR do sistema, dada por (4). Quanto menor $\mathrm{B}_{\mathrm{F}}$, maior a filtragem espectral das componentes FWM e melhor a SNR. No caso $\mathrm{ES}$, o valor de $\mathrm{B}_{\mathrm{F}}$ não afeta a $\mathrm{SNR}$, pois produtos $\mathrm{FWM}$ que ocorrem na banda do sistema sempre coincidem com canais. No caso US-G, como os espaçamentos entre canais $\left(\Delta \mathrm{f}_{\mathrm{i}}, \mathrm{i}=1\right.$, $2, \ldots, \mathrm{N}-1$ ) são variáveis e produtos $\mathrm{FWM}$ podem coincidir com canais, não há um critério especifico para a escolha de $\mathrm{B}_{\mathrm{F}}$, que passa a depender da configuração do sistema. No caso US-OGR, para a escolha de $\mathrm{B}_{\mathrm{F}}$, foram efetuadas exaustivas simulações numéricas [6], incluindo OGRs de ordem até 132, que permitiram concluir que: i) as posições relativas de canais e produtos FWM dependem apenas do número de canais; ii) a separação espectral entre dois produtos FWM adjacentes é um múltiplo inteiro da largura de slot $\Delta \mathrm{f}$ e iii) a separação espectral entre um canal e cada um dos produtos FWM adjacentes a ele é $\Delta \mathrm{f}$. Esta condição assegura que, se existirem dois canais tais que o espaçamento entre eles é $\Delta \mathrm{f}$, então nenhum produto FWM será gerado entre esse par de canais. A partir dessas conclusões e em todas as simulações realizadas com o esquema US-OGR, emprega-se um filtro óptico com largura de banda $\mathrm{B}_{\mathrm{F}}=2 \Delta \mathrm{f}$, centrado em cada canal de entrada.

Para um melhor entendimento e visualização das limitações impostas pelos esquemas de alocação de canais ES e US, considera-se o exemplo de um sistema WDM com 24 canais $(0,7 \mathrm{~mW} /$ canal $)$ e fibra $\operatorname{DSF}(\mathrm{D}=0, \mathrm{dD} / \mathrm{d} \lambda=0,07$ $\mathrm{ps} / \mathrm{km}^{-\mathrm{nm}^{2}}$ ) com comprimento $\mathrm{L}=22,3 \mathrm{~km}$. Para os esquemas $\mathrm{ES}(\Delta \mathrm{f}=100 \mathrm{GHz})$; US-G $\left(\mathrm{f}_{\text {ref }}=50 \mathrm{GHz}, \Delta \mathrm{F}=10 \mathrm{GHz}\right)$ e US-
OGR $(\Delta \mathrm{f}=12,5 \mathrm{GHz})$, os valores calculados para largura de banda e SNR são listados na Tabela I, enquanto as potências dos canais de entrada e dos produtos FWM no final da fibra são ilustradas na Figura 1.

TABELA I

VALORES CALULADOS PARA LARGURA DE BANDA E SNR, PARA ESQUEMAS ES, US-G E US-OGR

\begin{tabular}{|c|c|c|}
\hline \hline Esquema & Largura de Banda $(\mathrm{nm})$ & SNR $(\mathrm{dB})$ \\
\hline ES & 18,41 & 13,82 \\
\hline US-G & 29,44 & $24,42\left(\mathrm{~B}_{\mathrm{F}}=25 \mathrm{GHz}\right)$ \\
& & $22,41\left(\mathrm{~B}_{\mathrm{F}}=50 \mathrm{GHz}\right)$ \\
\hline US-OGR & 41,43 & $24,4\left(\mathrm{~B}_{\mathrm{F}}=25 \mathrm{GHz}\right)$ \\
\hline \hline
\end{tabular}

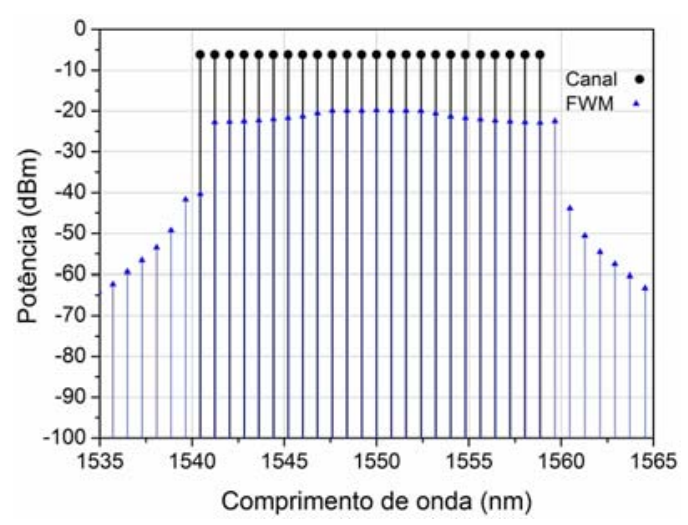

(a)

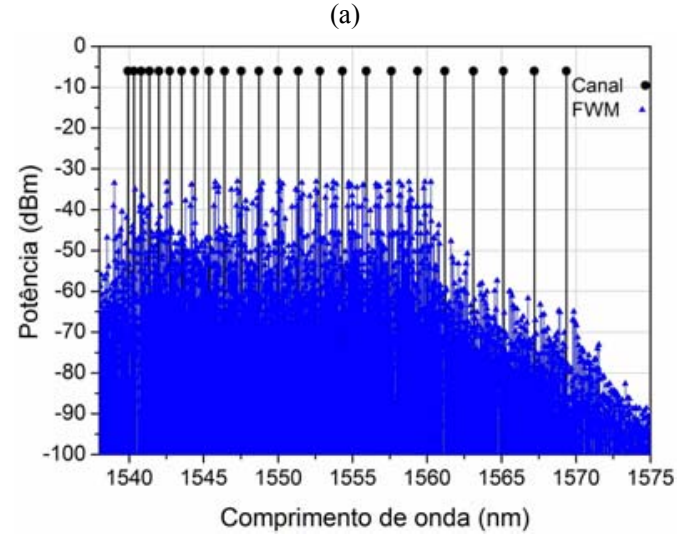

(b)

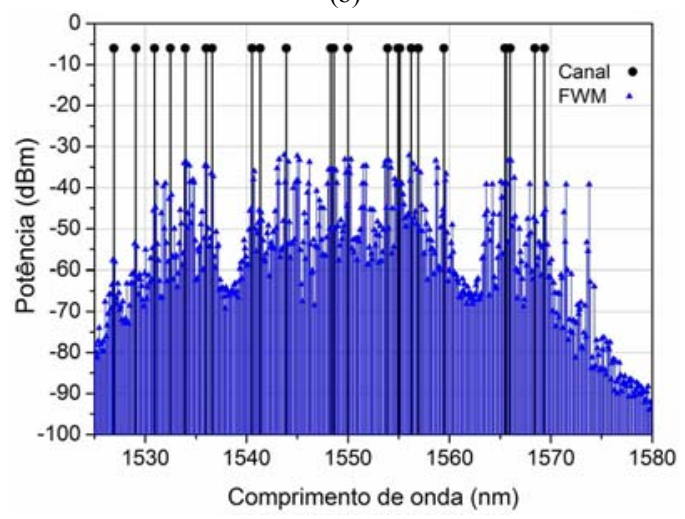

(c)

Fig. 1. Potências de canais e de FWM para um sistema de 24 canais, com alocação (a) $\mathrm{ES}(\Delta \mathrm{f}=100 \mathrm{GHz})$, (b) US-G $\left(\mathrm{f}_{\mathrm{ref}}=50 \mathrm{GHz}, \Delta \mathrm{F}=10 \mathrm{GHz}\right)$ e (c) US-OGR $(\Delta \mathrm{f}=12,5 \mathrm{GHz})$. 
A Figura 1 mostra que, no caso do esquema ES, a potência de FWM é praticamente constante em toda a largura de banda do sistema e que, nestes comprimentos de onda, produtos FWM sempre coincidem com canais de entrada. Nos casos dos esquemas US-G e US-OGR, observa-se uma redução da potência de FWM na largura de banda do sistema, em comparação com ES. Como esperado, as componentes mais intensas de FWM ocorrem nas vizinhanças do zero de dispersão da fibra DSF, mesmo no caso ES.

A Figura 1 também indica que os esquemas ES e USOGR apresentam limitações do ponto de vista prático: no primeiro, a SNR é insatisfatória e, no segundo, a largura de banda total ocupada pelos canais extrapola a faixa de operação adequada de EDFAs (banda C). Por sua vez, o esquema US-G, quando comparado com US-OGR, provê considerável redução da largura de banda, embora a manutenção de valores aceitáveis para a SNR dependa de uma escolha criteriosa da largura de banda dos filtros ópticos. A simulação de diversos outros casos mostrou que o aumento do número e/ou da potência dos canais torna o problema ainda mais crítico, sugerindo a necessidade da exploração de novos esquemas de alocação de canais.

A seguir, é apresentado um estudo comparativo de três modificações do esquema US-G.

\section{B. Espaçamento Não Uniforme Cíclico (USC)}

Este esquema, ilustrado na Figura 2, tem como característica a repetição cíclica de uma dada alocação US, denominada unidade de base de ordem $\mathrm{M}$, onde $\mathrm{M}$ designa o número de frequências nesta alocação US básica. A maior frequência de uma determinada unidade de base coincide com a menor frequência da unidade de base seguinte.

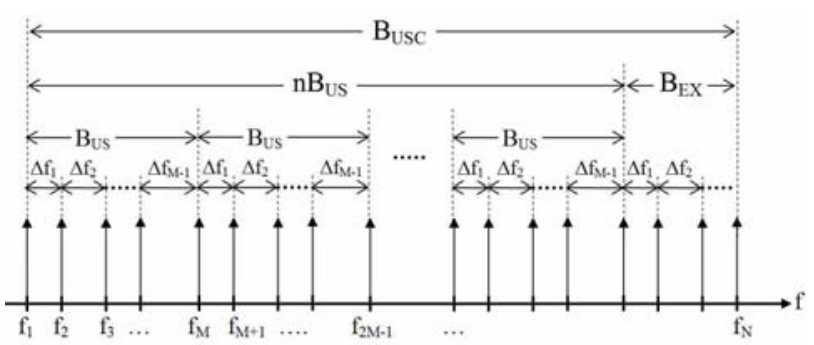

Fig. 2. Alocação de canais no esquema de espaçamento não uniforme cíclico (USC).

A largura de banda total ocupada pelo arranjo de canais com alocação USC é dada por $\mathrm{B}_{\mathrm{USC}}=\mathrm{nB}_{\mathrm{US}}+\mathrm{B}_{\mathrm{EX}}$, onde $\mathrm{n}$ é o número de unidades de base completamente utilizadas, $\mathrm{B}_{\mathrm{US}}$ é a largura de banda da unidade de base e $\mathrm{B}_{\mathrm{EX}}$ representa a largura de banda ocupada pelos canais excedentes.

Para exemplificar o esquema USC, considera-se uma unidade de base de ordem $M=4$ e do tipo US-G $\left(f_{\text {ref }}=100\right.$ $\mathrm{GHz}, \Delta \mathrm{F}=20 \mathrm{GHz}$ ). No caso de um sistema de 24 canais, são necessárias sete destas unidades de base completas (que acomodam 22 canais) e uma incompleta, para os dois canais restantes. Desta forma, as correspondentes frequências dos canais (em GHz) são: $(0,100,220, \mathbf{3 6 0}, 460,580,720, \ldots$, 2380, 2520, 2620, 2740). Os números em negrito representam a maior frequência de uma unidade de base e a menor frequência da unidade de base seguinte.

A largura de banda total é $\mathrm{B}_{\mathrm{USC}}=21,92 \mathrm{~nm}$ (ou 2740 $\mathrm{GHz}$ ). Os filtros ópticos utilizados têm larguras de 25 e $50 \mathrm{GHz}$ e os respectivos valores de SNR foram calculados como 20,85 e $18,10 \mathrm{~dB}$, e podem ser comparados com os da Tabela I. A utilização do esquema USC permite uma redução da largura de banda do sistema (em torno de $25 \%$, em relação à largura de banda do esquema US-G, e 53\% em relação ao esquema US-OGR), que é acompanhada de uma ligeira deterioração da $\mathrm{SNR}$. Isto significa um aumento do número de produtos FWM que coincidem com canais e/ou ocorrem na banda passante do filtro. A utilização de filtros ópticos mais seletivos mostrou ser possível melhorar a SNR apenas marginalmente. Outras simulações mostraram que existe um compromisso entre largura de banda e SNR satisfatórias: quanto menor a ordem da unidade de base $\mathrm{M}$, menor a largura de banda e pior a SNR. Para $\mathrm{M}=1$, por exemplo, recai-se no esquema ES. A seguir, são investigadas duas modificações do esquema USC.

\section{Espaçamento Não Uniforme Cíclico com Intervalo Uniforme (USC-U)}

Neste esquema, é introduzido um intervalo de frequência constante entre unidades de base adjacentes, como indicado na Figura 3.

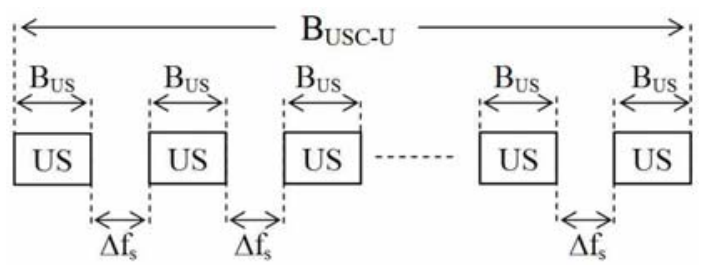

Fig. 3. Alocação de canais no esquema de espaçamento não uniforme cíclico com intervalo uniforme (USC-U).

Agora, a largura de banda total é dada por $\mathrm{B}_{\mathrm{USC}-\mathrm{U}}=\mathrm{nB}_{\mathrm{BUS}}$ $+(\mathrm{n}-1) \Delta \mathrm{f}_{\mathrm{s}}+\mathrm{B}_{\mathrm{EX}}$, onde $\mathrm{n}$ é o número de unidades de base completas, $\mathrm{B}_{\mathrm{US}}$ é a largura de banda da unidade de base, $\Delta \mathrm{f}_{\mathrm{s}}$ é o espaçamento constante entre unidades de base adjacentes e $\mathrm{B}_{\mathrm{EX}}$ é a largura de banda dos canais excedentes.

Embora possa parecer que este esquema resulta em maior largura de banda que o esquema USC, isto não é necessariamente verdade, pois agora todas as frequências nas unidades de base são utilizadas. Ou seja, um dado número de canais pode ser acomodado em um menor número de unidades de base. Por exemplo, os 24 canais considerados anteriormente podem ser acomodados em seis unidades de base de ordem $M$ $=4$. Para mesmos número de canais e $\mathrm{B}_{\mathrm{US}}$, a diferença entre $\mathrm{B}_{\text {USC-U }}$ e $\mathrm{B}_{\text {USC }}$ dependerá da escolha do intervalo $\Delta \mathrm{f}_{\mathrm{s}}$.

$\mathrm{O}$ esquema USC-U foi implementado, com $\Delta \mathrm{f}_{\mathrm{s}}=$ $110 \mathrm{GHz}$, para o sistema de 24 canais, unidade de base $(\mathrm{M}=$ 4) e fibra anteriores. As correspondentes frequências dos canais (em GHz) são: $(\underline{0}, 100,220,360, \underline{470}, 570,690, \mathbf{8 3 0}, \ldots$, $\underline{2350}, 2450,2570,2710)$. As frequências iniciais e finais de cada unidade de base são indicadas por números sublinhados e em negrito, respectivamente. Neste caso, a largura de banda total é de 21,68 nm (ou $2710 \mathrm{GHz}$ ). Os valores obtidos para SNR são: 17,22 dB, com filtro de $50 \mathrm{GHz}$, e 18,61 dB, com 
filtro de $25 \mathrm{GHz}$, e $21 \mathrm{~dB}$, com filtro de $12,5 \mathrm{GHz}$. Em comparação com os resultados do esquema USC, observa-se uma redução de $30 \mathrm{GHz}(0,24 \mathrm{~nm})$ na largura de banda do sistema e uma pequena deterioração da SNR.

\section{Espaçamento Não Uniforme Cíclico com Intervalo Não Uniforme (USC-NU)}

Neste esquema, é introduzido um intervalo de frequencia variável entre unidades de base adjacentes, como indicado na Figura 4.

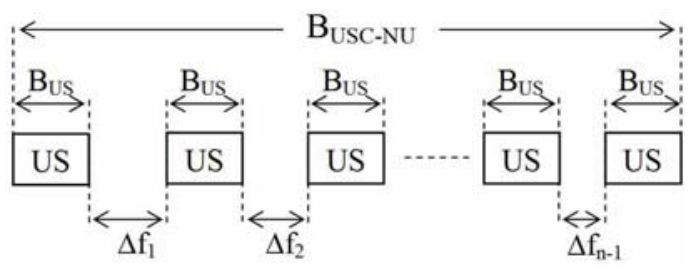

Fig. 4. Alocação de canais no esquema de espaçamento não uniforme cíclico com intervalo não uniforme (USC-NU).

A largura de banda total é dada por: $\mathrm{B}_{\mathrm{USC}-\mathrm{NU}}=\mathrm{nB}_{\mathrm{US}}+$

$\sum_{i=1}^{n-1} \Delta f_{i}+B_{E X}$, onde $n, B_{U S}$ e $B_{E X}$ têm os mesmos significados que no esquema USC-U. Para minimizar a largura de banda total, o espaçamento total $\Delta \mathrm{f}_{\mathrm{d}}=\sum_{\mathrm{i}=1}^{\mathrm{n}-1} \Delta \mathrm{f}_{\mathrm{i}}$ deve ser tão pequeno quanto possível. Obviamente, se $\Delta \mathrm{f}_{\mathrm{d}}<(\mathrm{n}-1) \Delta \mathrm{f}_{\mathrm{s}}$, sendo $\Delta \mathrm{f}_{\mathrm{s}} \mathrm{o}$ intervalo constante usado no esquema USC-U, a largura de banda $\mathrm{B}_{\mathrm{USC}-\mathrm{NU}}$ será menor que $\mathrm{B}_{\mathrm{USC}-\mathrm{U}}$, para mesmos $\mathrm{n}, \mathrm{B}_{\mathrm{US}} \mathrm{e}$ número de canais, $\mathrm{N}$.

Para efeitos de comparação, o esquema USC-NU foi aplicado ao sistema de 24 canais com a mesma unidade de base $(\mathrm{M}=4)$ e fibra já consideradas. Neste caso, os 24 canais também são acomodados em seis unidades de base, entre as quais foram inseridos os seguintes espaçamentos (em GHz): $\Delta \mathrm{f}_{1}=115, \Delta \mathrm{f}_{2}=110, \Delta \mathrm{f}_{3}=105, \Delta \mathrm{f}_{4}=95$ e $\Delta \mathrm{f}_{5}=90 \mathrm{GHz}$. As correspondentes frequências dos canais (em GHz) são: (므, $100,220, \mathbf{3 6 0}, \underline{475}, 575,695, \mathbf{8 3 5}, \ldots, \underline{2315}, 2415,2535, \mathbf{2 6 7 5})$. Novamente, as frequências iniciais e finais de cada unidade de base são indicadas por números sublinhados e em negrito, respectivamente.

A largura de banda total é de 21,4 nm (ou $2675 \mathrm{GHz}$ ). Os valores obtidos para SNR são: $17,84 \mathrm{~dB}$, com filtro de $50 \mathrm{GHz}$, e 20,10 dB, com filtro de $25 \mathrm{GHz}$. Comparando estes resultados com os do esquema USC-U, observa-se uma redução na largura da banda total de $35 \mathrm{GHz}$ (ou 0,28 nm) e uma melhora de cerca de $1,5 \mathrm{~dB}$ na SNR obtida com filtro de $25 \mathrm{GHz}$. A Tabela II compara os principais resultados associados aos esquemas USC, USC-U e USC-NU.

Os valores listados na Tabela II sugerem que o esquema USC-NU é um bom candidato para a alocação de comprimentos de onda em sistemas WDM, pois oferece a menor largura de banda e permite a obtenção de boa SNR, indicando uma redução na ocorrência de produtos FWM na banda passante do filtro óptico, em comparação com os outros esquemas US. A Tabela II também indica a importância da especificação da largura de banda do filtro de canal, pois a mesma afeta diretamente a SNR.

TABELA II

VALORES CALCULADOS PARA LARGURA DE BANDA E SNR, ESQUEMAS USC, USC-U E USC-NU

\begin{tabular}{|c|c|c|}
\hline \hline Esquema & Largura de Banda $(\mathrm{nm})$ & SNR $(\mathrm{dB})$ \\
\hline USC & 21,92 & $20,85\left(\mathrm{~B}_{\mathrm{F}}=25 \mathrm{GHz}\right)$ \\
& & $18,10\left(\mathrm{~B}_{\mathrm{F}}=50 \mathrm{GHz}\right)$ \\
\hline USC-U & 21,68 & $21,00\left(\mathrm{~B}_{\mathrm{F}}=12,5 \mathrm{GHz}\right)$ \\
& & $18,61\left(\mathrm{~B}_{\mathrm{F}}=25 \mathrm{GHz}\right)$ \\
& & $17,22\left(\mathrm{~B}_{\mathrm{F}}=50 \mathrm{GHz}\right)$ \\
\hline USC-NU & 21,40 & $20,10\left(\mathrm{~B}_{\mathrm{F}}=25 \mathrm{GHz}\right)$ \\
& & $17,84\left(\mathrm{~B}_{\mathrm{F}}=50 \mathrm{GHz}\right)$ \\
\hline \hline
\end{tabular}

A seguir, é explorada a influência da largura de banda do filtro óptico.

\section{INFLUÊNCIA DO FILTRO ÓPTICO}

Visando analisar a influência do filtro óptico na SNR, foi calculado, para o sistema de 24 canais considerado na seção anterior, o número de produtos FWM que ocorrem na banda passante do filtro. No esquema ES, a influência dos filtros ópticos não é observada, independentemente do espaçamento entre canais, pois todos os produtos FWM que ocorrem na banda do sistema coincidem com canais de entrada. No esquema US-OGR, são considerados filtros ópticos com $\mathrm{B}_{\mathrm{F}}=2 \Delta \mathrm{f}$, como já comentado. Nos casos dos esquemas USC, USC-U e USC-NU, são considerados filtros com bandas passantes de 50, 25, 12,5 e 6,25 GHz. Vale a pena ressaltar que, no esquema US-OGR, o filtro óptico de $50 \mathrm{GHz}$ (correspondendo a um slot de $\Delta \mathrm{f}=25 \mathrm{GHz}$ ) não foi utilizado, o que acarretaria considerável aumento na largura de banda. Os resultados são mostrados na Figura 5.
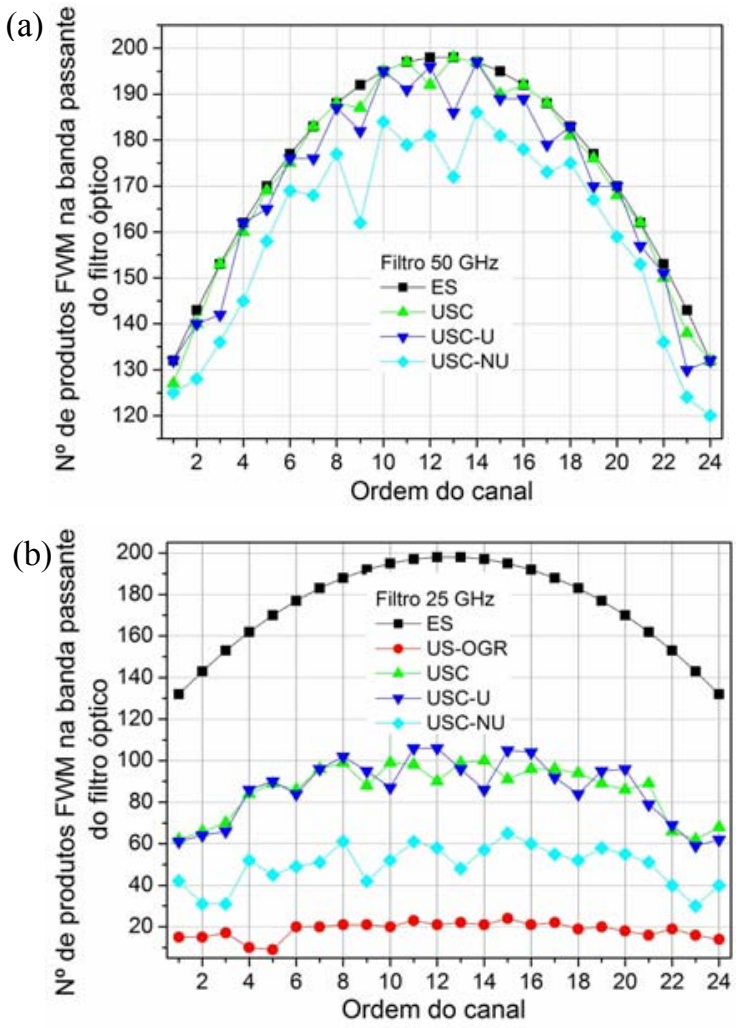
(c)

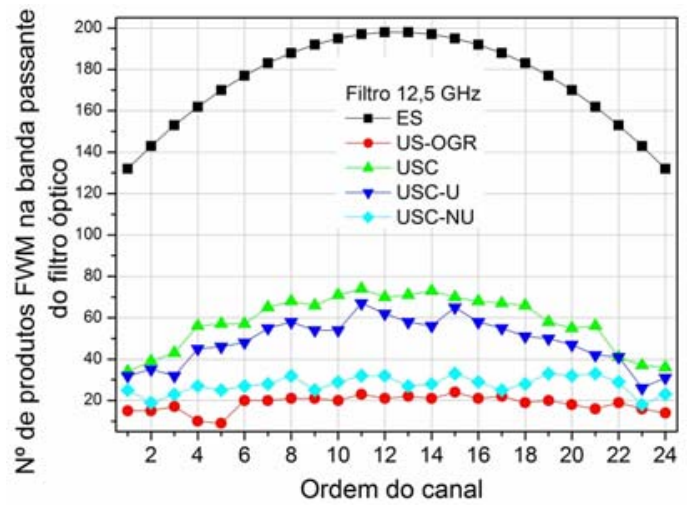

(d)

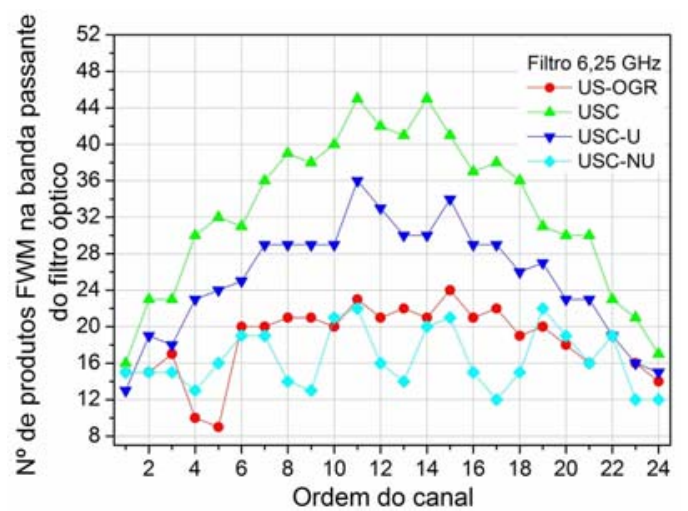

Fig. 5. Número de produtos FWM na banda passante do filtro óptico em função da ordem do canal para os esquemas ES, US-OGR, USC, USC-U e USC-NU e filtros ópticos de largura: (a) 50; (b) 25; (c) 12,5 e (d) 6,25 GHz.

O canal 12 (canal central) está posicionado no comprimento de onda de zero de dispersão da fibra DSF $\left(\lambda_{\mathrm{ZD}}=\right.$ $1550 \mathrm{~nm}$ ). Como esperado, as curvas correspondendo ao esquema ES são idênticas, confirmando que o filtro óptico não reduz o número de produtos FWM na banda do canal. Outro fato aparente é que, independentemente do esquema de alocação de canais, há geração de um grande número de produtos FWM nas vizinhanças de $\lambda_{Z D}$ e, portanto, maior interferência nos canais centrais do que nos canais laterais (mais afastados de $\lambda_{\mathrm{ZD}}$ ).

Na Figura 5(a), observa-se claramente que as curvas dos esquemas US têm comportamentos bastante semelhantes ao de ES: maior concentração de produtos FWM em torno dos canais centrais com rápida redução em torno dos canais laterais. Esse caso mostra que a filtragem de produtos FWM é pequena e praticamente independe do esquema de alocação de canais, fato confirmado por valores semelhantes para SNR, de acordo com a Tabela II, com $\mathrm{B}_{\mathrm{F}}=50 \mathrm{GHz}$. O esquema US-OGR, quando comparado com os outros esquemas US, possui um menor número de produtos FWM gerados na banda do filtro óptico, que é praticamente constante, em torno de 20, para todos os canais. Nesse caso, a eficiência da filtragem espectral resulta da escolha de $\mathrm{B}_{\mathrm{F}}=2 \Delta \mathrm{f}$. Apenas com o filtro de 6,25 GHz (Figura 5(d)), o esquema USC-NU mostra-se superior ao esquema USOGR. Vale lembrar, no entanto, que as larguras de banda associadas ao esquema US-OGR são excessivamente grandes.

De um modo geral, o esquema USC-NU é o que resulta em menor número de produtos FWM na banda passante do filtro, seguido pelos esquemas USC-U e USC. Os resultados confirmam o esquema USC-NU como o mais adequado para a alocação de canais em sistemas WDM, pois provê bom compromisso entre largura de banda total e SNR, considerando filtros ópticos de 25 e 12,5 GHz. Melhorias na SNR são obtidas para filtros de $6,25 \mathrm{GHz}$.

\section{CONCLUSÕES}

Este trabalho apresentou um estudo comparativo de como diferentes esquemas de alocação de canais baseados em espaçamento não uniforme (US): US-G, US-OGR, USC, USCU, USC-NU, afetam a geração de FWM em sistemas ópticos multicanal com fibra de dispersão deslocada. A influência da banda passante de filtros ópticos também foi investigada. $\mathrm{O}$ modelo utilizado supõe bombeio sem depleção e permite avaliar o descasamento de fase, potência de FWM e a SNR do sistema. A largura de banda e a SNR do sistema são usadas como figuras de mérito. De um modo geral, o esquema USOGR provê a melhor SNR, mas às custas de um aumento da largura de banda do sistema, por vezes proibitivo. Os resultados mostram, ainda, que o esquema USC-NU provê bom compromisso entre largura de banda $(\sim 20 \mathrm{~nm})$ e SNR $(>20$ $\mathrm{dB}$ ), considerando um sistema WDM de 24 canais com filtro óptico de $25 \mathrm{GHz}$. Outras simulações, utilizando filtros mais seletivos, de 12,5 e $6,25 \mathrm{GHz}$, indicaram que eles têm papel fundamental no desempenho dos sistemas, melhorando a SNR, em particular naqueles com elevado número de canais.

\section{REFERÊNCIAS}

[1] G. P. Agrawal, "Nonlinear Fiber Optics" ( $4^{\text {th }}$ ed.), Academic Press, San Diego, New York, USA, 2006.

[2] P. B. Harboe, E. da Siva e J. R. Souza, "Simulador WDM para Análise das Penalidades Impostas por FWM em Fibras G.652, G.653 e G.655", aceito para publicação no MOMAG2008, Florianópolis-SC, Setembro 2008.

[3] P. B. Harboe, E. da Siva e J. R. Souza, "FWM em Sistemas WDM: Espaçamento Não Uniforme versus Espaçamento Uniforme", aceito para publicação no XXVI Simpósio Brasileiro de Telecomunicações (SBrT'08), Rio de Janeiro-RJ, Setembro 2008.

[4] P. B. Harboe, E. da Silva, and J. R. Souza, "Analysis of FWM Penalties in DWDM Systems Based on G.652, G.653, and G.655 Optical Fibers", In: WCSET 2008: World Congress on Science, Engineering and Technology, 2008, Bangkok. Proceedings of World Academy of Science, Engineering and Technology, v 36, 2008.

[5] P. B. Harboe and J. R. Souza, "Analysis of Four-Wave Mixing in Optical Fiber Links with Non-Uniform Chromatic Dispersion”, Microwave and Optical Tech. Letters, v 39, n 2, pp. 102-105, 2003.

[6] E. da Silva, "Análise de Penalidades Impostas por FWM em Sistemas WDM", Dissertação de Mestrado apresentada ao Departamento de Engenharia de Telecomunicações, UFF, Niterói- RJ, 2008.

[7] J. Lawson, Projeto Distributed.net, www.distributed.net, visitado em $02 / 05 / 2009$.

[8] J. Onishi, S. Kojima, and T. Numai, "Effects of Frequency Allocations and Zero-Dispersion Frequencies on FDM Lightwave Transmission Systems", Journal of Ligthwave Tech., v 25, n 7, pp. 1719-1727, 2007.

[9] Y. Nagatani et all, "Theoretical Analysis of Frequency Allocations FDM Lightwave Transmission Systems", Journal of Ligthwave Tech., v 26, n 13, pp. 1993-2001, 2008.

[10] K. Nakajima, M. Ohashi, Y. Miyajima, and K. Shiraki, "Assessment of dispersion varying fibre in WDM system”, Electronics Letters, v 33, n 12, pp. 1059-1060, 1997. 